\title{
Variational-Like Inequalities for Weakly Relaxed $\eta-\alpha$ Pseudomonotone Set-Valued Mappings in Banach Space
}

\author{
Syed Shakaib Irfan and Mohammad Firdosh Khan \\ College of Engineering, Qassim University, P.O. Box 6677, Buraidah, Al-Qassim 51452, Saudi Arabia \\ Correspondence should be addressed to Syed Shakaib Irfan; shakaib@qec.edu.sa
}

Received 1 July 2016; Accepted 18 August 2016

Academic Editor: Julien Salomon

Copyright ( 92016 S. S. Irfan and M. F. Khan. This is an open access article distributed under the Creative Commons Attribution License, which permits unrestricted use, distribution, and reproduction in any medium, provided the original work is properly cited.

We introduce and study variational-like inequalities for generalized pseudomonotone set-valued mappings in Banach spaces. By using KKM technique, we obtain the existence of solutions for variational-like inequalities for generalized pseudomonotone setvalued mappings in reflexive Banach spaces. The results presented in this paper are generalizations and improvements of the several well-known results in the literature.

\section{Introduction and Preliminaries}

Variational inequality theory plays an important role in many fields, such as optimal control, mechanics, economics, transportation equilibrium, and engineering science. It is well known that monotonicity plays an important role in the study of variational inequality theory. In recent years, a number of authors have proposed many generalizations of monotonicity such as pseudomonotonicity, relaxed monotonicity, relaxed $\eta-\alpha$ monotonicity, quasimonotonicity, and semimonotonicity, $p$-monotonicity. For details, refer to [1-11] and the references therein.

Verma [11] studied a class of variational inequalities with relaxed monotone operators. In 2003, Fang and Huang [4] introduced a new concept of relaxed $\eta-\alpha$ monotonicity and obtained the existence of solution for variational-like inequalities in reflexive Banach spaces. B. S. Lee and B. D. Lee [12] defined weakly relaxed $\alpha$-semipseudomonotone setvalued variational-like inequalities and generalize the result of Fang and Huang [4]. Bai et al. [1] defined relaxed $\eta$ $\alpha$-pseudomonotone concepts for single valued mappings. For set-valued mappings, Kang et al. [13] defined relaxed $\eta$ - $\alpha$ pseudomonotone concepts which generalize monotone concepts for single valued mapping in Fang and Huang [4] and Bai et al. [1]. Recently Sintunavarat [14] established the existence of solution of mixed equilibrium problem with the weakly relaxed $\alpha$-monotone bifunction in Banach spaces.
In 2013, Kutbi and Sintunavarat [15] introduce two new concepts of weakly relaxed $\eta-\alpha$ monotone mappings and weakly relaxed $\eta$ - $\alpha$ semimonotone mappings and obtained the existence of solution for variational-like inequality problems in reflexive Banach spaces.

Inspired and motivated by the results of Fang and Huang [4] and Kutbi and Sintunavarat [15], in this paper, we introduce the concept of weakly relaxed $\eta-\alpha$ pseudomonotone mapping and by using Knaster Kuratowski Mazurkiewicz (KKM) technique [16], we study some existence of solution for variational-like inequality for set-valued pseudomonotone mapping.

In this paper, we suppose that $E$ is a reflexive Banach space with dual space $E^{*}$, and $\langle\cdot, \cdot\rangle$ denotes the pairing between $E$ and $E^{*}$. Let $K$ be a nonempty closed convex subset of $E$ and $2^{E}$ denote the family of all the nonempty subset of $E$.

The following definitions and results will be useful in our work.

Definition 1. A mapping T $K \rightarrow 2^{E^{*}}$ is said to be weakly relaxed $\eta-\alpha$ pseudomonotone if there exist a mapping $\eta: K \times$ $K \rightarrow E$ and functions $\phi: K \times K \rightarrow R \cup\{+\infty\}, \alpha: E \rightarrow R$ with $\alpha(t, z)=k(t) \alpha(z)$ for $z \in E$, where $k:(0, \infty) \rightarrow(0, \infty)$ is a function with $\lim _{t \rightarrow 0}(k(t) / t)=0$, such that

$$
\langle u, \eta(x, y)\rangle+\phi(y, x)-\phi(x, x) \geq 0
$$


implying

$$
\begin{aligned}
& \langle v, \eta(x, y)\rangle+\phi(y, x)-\phi(x, x) \geq \alpha(x-y) \\
& \quad \text { for } x, y \in E, u \in T(x), v \in T(y)
\end{aligned}
$$

Remark 2. (i) If $\phi(x, x)=0$ in Definition 1 then we have the following pseudomonotone concept defined in Kang et al. [13]:

$$
\langle u, \eta(y, x)\rangle+\phi(y, x) \geq 0 \quad \forall u \in T(x),
$$

implying

$$
\langle v, \eta(y, x)\rangle+\phi(y, x) \geq \alpha(y-x), \quad \forall v \in T(y) .
$$

(ii) If $T$ is single valued mappings, $\phi(x, x)=0$, and $k(t)=t^{p}$ for $p>1$, then we have the following relaxed $\eta^{-}$ $\alpha$ monotone concepts defined in Fang and Huang [4] and the following $\eta-\alpha$ pseudomonotone concepts, defined in Bai et al. [1]:

(a) For any $x, y \in K$

$$
\langle T x-T y, \eta(x, y)\rangle \geq \alpha(x-y) .
$$

(b) For any $x, y \in K$

$$
\begin{gathered}
\langle T y, \eta(x, y)\rangle \geq 0 \\
\text { implies }\langle T x, \eta(x, y)\rangle \geq \alpha(x-y) .
\end{gathered}
$$

Definition 3 (see [17]). Let $T: K \rightarrow 2^{E^{*}}$ and $\eta: K \times K \rightarrow K$ be two mappings; $T$ is said to be $\eta$-hemicontinuous for any $x, y \in K$, if the mapping defined by $\phi:[0,1] \rightarrow \mathbb{R}$ defined by

$$
\phi(t)=\langle T(t y+(1-t) x), \eta(y, x)\rangle
$$

is continuous at $0^{+}$.

Definition 4 (see [4]). Let $T: K \rightarrow 2^{E^{*}}$ and $\eta: K \times K \rightarrow K$ be two mappings and $\phi: K \times K \rightarrow \mathbb{R} \bigcup\{+\infty\}$ be a proper functional. Then $T$ is said to be $\eta$-coercive with respect to first argument of $\phi$, if there exists $x_{0} \in K$ such that

$$
\frac{\left\langle u-u_{0}, \eta\left(x, x_{0}\right)\right\rangle+\phi\left(x, x_{0}\right)-\phi\left(x_{0}, x_{0}\right)}{\left\|\eta\left(x, x_{0}\right)\right\|} \longrightarrow \infty,
$$

whenever $\|x\| \rightarrow \infty$, for all $u \in T(x), u_{0} \in T\left(x_{0}\right)$.

If $\phi(\cdot, \cdot)=\phi(\cdot)$ then there exists $x_{0} \in K$ such that

$$
\frac{\left\langle u-u_{0}, \eta\left(x, x_{0}\right)\right\rangle+\phi(x)-\phi\left(x_{0}\right)}{\left\|\eta\left(x, x_{0}\right)\right\|} \longrightarrow \infty,
$$

whenever $\|x\| \rightarrow \infty$, for all $u \in T(x), u_{0} \in T\left(x_{0}\right)$.

If $\phi=\delta_{K}$, where $\delta_{K}$ is the indicator function of $K$, then Definition 4 coincides with the definition of $\eta$-coercivity in the sense of Yang and Chen [18].
Definition 5. A multivalued mapping $T: K \rightarrow 2^{E^{*}}$ is said to be relaxed $\eta-\alpha$ monotone if there exists a function $\eta: K \times K \rightarrow$ $E$ and $\alpha: E \rightarrow E$ with $\alpha(t z)=t^{p} \alpha(z)$ for all $t>0, p>1$, and $z \in E$ such that

$$
\begin{aligned}
\langle u-v, \eta(x, y)\rangle \geq & \alpha(x-y), \\
& \forall x, y \in K, u \in T(x), v \in T(y) .
\end{aligned}
$$

Remark 6. (i) If $T$ is single valued then (10) becomes

$$
\langle T x-T y, \eta(x, y)\rangle \geq \alpha(x-y), \quad \forall x, y \in K,
$$

and then $T$ is said to be relaxed $\eta-\alpha$ monotone [4].

(ii) If $T$ is single valued and $\eta(x, y)=x-y$ then (10) becomes

$$
\langle T x-T y, x-y\rangle \geq \alpha(x-y), \quad \forall x, y \in K,
$$

and then $T$ is called relaxed $\alpha$-monotone [4].

(iii) If $\eta(x, y)=x-y$ for all $x, y \in K$ and $\alpha(z)=k\|z\|^{p}$, where $k>0$ and $p>1$, then (12) becomes

$$
\langle T x-T y, x-y\rangle \geq k\|x-y\|^{p}, \quad \forall x, y \in K,
$$

and $T$ is called $p$-monotone $[5,10,11]$.

Definition 7. A mapping $T: K \rightarrow 2^{E}$ is said to be weakly relaxed $\eta$ - $\alpha$ monotone if there exists a function $\eta: K \times K \rightarrow E$ and $\alpha: E \rightarrow \mathbb{R}$ with

$$
\begin{aligned}
\lim _{t \rightarrow 0^{+}} \alpha(t x) & =0, \\
\lim _{t \rightarrow 0^{+}} \frac{d}{d t} \alpha(t x) & =0
\end{aligned}
$$

for all $t>0$ and $x \in E$ such that

$$
\begin{aligned}
\langle u-v, \eta(x, y)\rangle \geq & \alpha(x-y), \\
& \forall x, y \in E, u \in T(x), v \in T(y) .
\end{aligned}
$$

Remark 8. If $T$ is single valued mapping then Definition 7 reduces to Definition 9 of [15].

Remark 9. If $T$ is weakly relaxed $\eta$ - $\alpha$ monotone, then $T$ is weakly relaxed $\eta-\alpha$ pseudomonotone mapping but the converse is not true.

Definition 10 (see [16]). A mapping $F: K \rightarrow 2^{E}$ is said to be KKM mapping if, for any $\left\{x_{1}, \ldots, x_{n}\right\} \subset K, \operatorname{co}\left\{x_{1}, \ldots, x_{n}\right\} \subset$ $\bigcup_{i=1}^{n} F\left(x_{i}\right)$, where $\operatorname{co}\left\{x_{1}, \ldots, x_{n}\right\}$ denote the convex hull of $x_{1}, \ldots, x_{n}$.

Lemma 11 (see [19]). Let $M$ be a nonempty subset of $a$ Hausdorff topological vector space $X$ and let $F: M \rightarrow 2^{X}$ be a KKM mapping. If $F(x)$ is closed in $X$ for all $x \in M$ and compact for some $x \in M$, then

$$
\bigcap_{x \in M} F(x) \neq \phi .
$$




\section{Existence Results}

In this section, we discuss the existence of the following variational-like inequality:

Find $x \in K$ such that

$$
\begin{aligned}
\langle u, \eta(y, x)\rangle+\phi(y, x)-\phi(x, x) & \geq 0, \\
\forall y & \in K, u \in T(x),
\end{aligned}
$$

where $K$ is a nonempty closed convex subset of a reflexive Banach space $E$.

Theorem 12. Suppose that $T: K \rightarrow 2^{E^{*}}$ is $\eta$-hemicontinuous and weakly relaxed $\eta$ - $\alpha$ pseudomonotone mapping. Let $\phi: K \times$ $K \rightarrow \mathbb{R} \bigcup\{+\infty\}$ be a proper convex function and $\eta: K \times K \rightarrow$ $E$ be a mapping. Suppose that the following conditions hold:

(i) $\eta(x, x)=0, \forall x \in K$.

(ii) For any fixed $y \in K, u \in T(x)$, the mapping $x \rightarrow\langle u$, $\eta(x, y)\rangle$ is convex.

(iii) $x \rightarrow \eta(x, \cdot)$ and $x \rightarrow f(x, \cdot)$ are convex.

Then problems (16) and (17) are equivalent as follows:

Find $x \in K$ such that

$$
\langle u, \eta(y, x)\rangle+\phi(y, x)-\phi(x, x) \geq 0,
$$

$$
\forall y \in K, u \in T(x),
$$

Find $x \in K$ such that

$$
\begin{aligned}
\langle v, \eta(y, x)\rangle+\phi(y, x)-\phi(x, x) & \geq \alpha(y-x), \\
& \forall y \in K, v \in T(y) .
\end{aligned}
$$

Proof. Suppose that (16) has a solution. So there exist $x \in K$

$$
\begin{aligned}
\langle u, \eta(y, x)\rangle+\phi(y, x)-\phi(x, x) & \geq 0, \\
& \forall y \in K, u \in T(x) .
\end{aligned}
$$

Since $T$ is weakly relaxed $\eta-\alpha$ pseudomonotone, we have

$$
\begin{aligned}
\langle v, \eta(y, x)\rangle+\phi(y, x)-\phi(x, x) & \geq \alpha(y-x), \\
& \forall y \in K, \quad v \in T(y) .
\end{aligned}
$$

Therefore $x \in K$ is a solution of (17).

Conversely, suppose that $x \in K$ is a solution of (17) and $y \in K$ is any point with $\phi(y, y)<\infty$. From (17) we know that $\phi(x, x)<\infty$. For $t \in(0,1)$ let $y_{t}=(1-t) x+t y, t \in(0,1)$; then we have $y_{t} \in K$. Since $x \in K$ is a solution of problem (17), it follows that

$$
\begin{aligned}
& \left\langle v_{t}, \eta\left(y_{t}, x\right)\right\rangle+\phi\left(y_{t}, x\right)-\phi(x, x) \geq \alpha\left(y_{t}-x\right) \\
& =\alpha(t(y-x))=k(t) \alpha(y-x) .
\end{aligned}
$$

The convexity of $\phi$ and condition (ii) of Theorem 12 imply that

$$
\begin{aligned}
\phi\left(y_{t}, x\right)-\phi(x, x) \geq & \phi((1-t) x+t y, x)-\phi(x, x) \\
\leq & t(\phi(y, x)-\phi(x, x)), \\
\left\langle v_{t}, \eta\left(y_{t}, x\right)\right\rangle= & \left\langle v_{t}, \eta((1-t) x+t y, x)\right\rangle \\
\leq & (1-t)\left\langle v_{t}, \eta(x, x)\right\rangle \\
& +t\left\langle v_{t}, \eta(y, x)\right\rangle \\
= & t\left\langle v_{t}, \eta(y, x)\right\rangle .
\end{aligned}
$$

It follows from (21) that

$$
\begin{aligned}
\left\langle v_{t},\right. & \eta(y, x)\rangle+\phi(y, x)-\phi(x, x) \geq \frac{\alpha(t(y-x))}{t} \\
& =\frac{k(t)}{t} \alpha(y-x)
\end{aligned}
$$

for all $y \in K$ and $v_{t} \in T\left(y_{t}\right)$. Taking $t \rightarrow 0^{+}$in the previous inequality and using $\eta$-hemicontinuity of $T$, we get

$$
\langle u, \eta(y, x)\rangle+\phi(y, x)-\phi(x, x) \geq 0,
$$

for all $y \in K$ and $u \in T(x)$ with $\phi(y, y)<\infty$. In case of $\phi(y, y)=\infty$ the relation

$$
\langle u, \eta(y, x)\rangle+\phi(y, x)-\phi(x, x) \geq 0
$$

is trivial. Therefore $x \in K$ is solution of (16).

Theorem 13. Let $K$ be a nonempty bounded closed convex subset of a real reflexive Banach space $E$ and $E^{*}$ the dual space of E. Suppose that $T: \mathrm{K} \rightarrow 2^{E^{*}}$ is an $\eta$-hemicontinuous and weakly relaxed $\eta$ - $\alpha$ pseudomonotone mapping. Let $\phi: K \times K \rightarrow$ $\mathbb{R} \bigcup\{+\infty\}$ be a proper convex lower semicontinuous function and $\eta: K \times K \rightarrow E$ be a mapping. Assume that

(i) $\eta(x, y)+\eta(y, x)=0, \forall x \in K$,

(ii) for any fixed $y \in K$ and $u \in T(x)$ the mapping $x \mapsto\langle u, \eta(y, x)\rangle$ is convex and lower semicontinuous function,

(iii) $x \rightarrow \eta(x, \cdot)$ and $x \rightarrow f(x, \cdot)$ are convex and lower semicontinuous,

(iv) $\alpha: E \rightarrow \mathbb{R}$ is weakly lower semicontinuous; that is, for any net $\left\{x_{\beta}\right\}, x_{\beta}$ converges to $x$ in $\sigma\left(E, E^{*}\right)$ implying that $\alpha(x) \leq \lim \inf \alpha\left(x_{\beta}\right)$.

Then problem (17) is solvable.

Proof. Define two set-valued mappings $F, G: K \rightarrow 2^{E}$ as follows:

$$
\begin{aligned}
& F(y)=\{x \in K: \exists u \in T(x),\langle u, \eta(y, x)\rangle+\phi(y, x) \\
& \quad-\phi(x, x) \geq 0\} \quad \forall y \in K, \\
& G(y)=\{x \in K: \exists v \in T(y),\langle v, \eta(y, x)\rangle+\phi(y, x) \\
& \quad-\phi(x, x) \geq \alpha(y-x)\} \quad \forall y \in K .
\end{aligned}
$$


We claim that $F$ is a KKM mapping. If $F$ is not a KKM mapping, then there exist $\left\{y_{1}, y_{2}, \ldots, y_{n}\right\} \subset K$ such that $\operatorname{co}\left\{y_{1}, y_{2}, \ldots, y_{n}\right\} \nsubseteq \bigcup_{i=1}^{n} F\left(y_{i}\right)$. This implies that there exist $y_{0} \in \operatorname{co}\left\{y_{1}, y_{2}, \ldots, y_{n}\right\}$ such that $y=\sum_{i=1}^{n} t_{i} y_{i}$, where $t_{i} \geq$ $0, i=1,2, \ldots, n$, and $\sum_{i=1}^{n} t_{i}=1$, but $y \notin \bigcup_{i=1}^{n} F\left(y_{i}\right)$. From the definition of $F$, we have

$$
\begin{aligned}
&\left\langle v, \eta\left(y_{i}, y\right)\right\rangle+\phi\left(y_{i}, y\right)-\phi(y, y)< \\
& \qquad \text { for } i=1,2, \ldots, n,
\end{aligned}
$$

and it follows that

$$
\begin{aligned}
0 & =\langle v, \eta(y, y)\rangle=\left\langle v, \eta\left(\sum_{i=1}^{n} t_{i} y_{i}, y\right)\right\rangle \\
& \leq \sum_{i=1}^{n} t_{i}\left\langle v, \eta\left(y_{i}, y\right)\right\rangle<\sum_{i=1}^{n} t_{i}\left(\phi(y, y)-\phi\left(y_{i}, y\right)\right) \\
& =\phi(y, y)-\sum_{i=1}^{n} t_{i} \phi\left(y_{i}, y\right) \leq \phi(y, y)-\phi(y, y)=0,
\end{aligned}
$$

which is a contradiction. This implies that $F$ is a KKM mapping. Now we prove that $F(y) \subset G(y)$, for all $y \in K$.

For any given $y \in K$ and letting $x \in F(y)$, we have

$$
\langle u, \eta(y, x)\rangle+\phi(y, x)-\phi(x, x) \geq 0 .
$$

Since $T$ is weakly relaxed $\eta$ - $\alpha$ pseudomonotone, we get

$$
\langle v, \eta(y, x)\rangle+\phi(y, x)-\phi(x, x) \geq \alpha(y-x) .
$$

It follows that $x \in G(y)$ and so $F(y) \subset G(y)$.

This implies that $G$ is also KKM mapping. From the assumption, we know that $G(y)$ is weakly closed for all $y \in$ $K$. In fact since $x \mapsto\langle v, \eta(y, x)\rangle$ and $\phi$ are two convex lower semicontinuous functions, from the definition of $G$ and weakly semilower continuity of $\alpha$, it is easy to see that $G(y)$ is weakly closed for all $y \in K$. Since $K$ is bounded closed and convex, we know that $K$ is weakly compact and so $G(y)$ is weakly compact in $K$ for each $y$ in $K$. From Lemma 11 and Theorem 12, we obtain that

$$
\bigcap_{y \in K} F(y)=\bigcap_{y \in K} G(y) \neq \phi
$$

Hence, there exists $x \in K$ such that

$$
\begin{aligned}
&\langle u, \eta(y, x)\rangle+\phi(y, x)-\phi(x, x) \geq 0, \\
& \forall x \in K, u \in T(x) ;
\end{aligned}
$$

that is, problem (16) has a solution.

Theorem 14. Let $K$ be a nonempty unbounded closed convex subset of a real reflexive Banach space $E$ and $E^{*}$ the dual space of E. Suppose that $T: K \rightarrow 2^{E^{*}}$ is an $\eta$-hemicontinuous and weakly relaxed $\eta$ - $\alpha$ pseudomonotone mapping. Let $\phi: K \times K \rightarrow$ $\mathbb{R} \bigcup\{+\infty\}$ be a proper convex lower semicontinuous function and $\eta: K \times K \rightarrow E$ be a mapping. Assume that

(i) $\eta(x, y)+\eta(y, x)=0, \forall x \in K$, (ii) for any fixed $y \in K$ and $u \in T(x)$ the mapping $x \mapsto\langle u, \eta(y, x)\rangle$ is convex and lower semicontinuous function,

(iii) $x \rightarrow \eta(x, \cdot)$ and $x \rightarrow f(x, \cdot)$ are convex and lower semicontinuous,

(iv) $\alpha: E \rightarrow \mathbb{R}$ is weakly lower semicontinuous,

(v) $T$ is $\eta$-coercive with respect to $\phi$; that is, there exist $x_{0} \in$ K such that

$$
\frac{\left\langle u-u_{0}, \eta\left(x, x_{0}\right)\right\rangle+\phi\left(x, x_{0}\right)-\phi\left(x_{0}, x_{0}\right)}{\left\|\eta\left(x, x_{0}\right)\right\|} \longrightarrow+\infty,
$$

$$
\text { whenever }\|x\| \rightarrow \infty \text {. }
$$

Then problem (16) is solvable.

Proof. Let

$$
B_{r}=\{y \in E:\|y\| \leq r\} .
$$

Consider the following problem, $x_{r} \in K \cap B_{r}$, such that

$$
\begin{aligned}
&\left\langle u_{r}, \eta\left(y, x_{r}\right)\right\rangle+\phi\left(y, x_{r}\right)-\phi\left(x_{r}, x_{r}\right) \geq 0, \\
& \forall y \in K \text { such that } y \in K \cap B_{r} .
\end{aligned}
$$

By Theorem 13, we know that (26) has a solution $x_{r} \in K \cap B_{r}$; choose $r>\left\|x_{0}\right\|$ with $x_{0}$ as in the coercivity conditions. Then we have

$$
\left\langle u_{r}, \eta\left(x_{0}, x_{r}\right)\right\rangle+\phi\left(x_{0}, x_{r}\right)-\phi\left(x_{r}, x_{r}\right) \geq 0 .
$$

Moreover

$$
\begin{aligned}
& \left\langle u_{r}, \eta\left(x_{0}, x_{r}\right)\right\rangle+\phi\left(x_{0}, x_{r}\right)-\phi\left(x_{r}, x_{r}\right)=-\left\langle u_{r}\right. \\
& \left.\quad-u_{0}, \eta\left(x_{r}, x_{0}\right)\right\rangle+\phi\left(x_{0}, x_{r}\right)-\phi\left(x_{r}, x_{r}\right) \\
& \quad+\left\langle u_{0}, \eta\left(x_{0}, x_{r}\right)\right\rangle \leq-\left\langle u_{r}-u_{0}, \eta\left(x_{r}, x_{0}\right)\right\rangle \\
& \quad+\phi\left(x_{0}, x_{r}\right)-\phi\left(x_{r}, x_{r}\right)+\left\|u_{0}\right\|\left\|\eta\left(x_{0}, x_{r}\right)\right\| \\
& \quad \leq\left\|\eta\left(x_{r}, x_{0}\right)\right\| \\
& \quad \cdot\left(\frac{\left\langle u_{r}-u_{0}, \eta\left(x_{r}, x_{0}\right)\right\rangle+\phi\left(x_{r}, x_{0}\right)-\phi\left(x_{0}, x_{0}\right)}{\left\|\eta\left(x, x_{0}\right)\right\|}\right. \\
& \left.\quad+\left\|u_{0}\right\|\right) .
\end{aligned}
$$

Now if $\|x\|=r$ for all $r$, we may choose $r$ large enough so that the above inequality and $\eta$-coercivity of $T$ with respect to $\phi$ imply that

$$
\left\langle u_{r}, \eta\left(x_{0}, x_{r}\right)\right\rangle+\phi\left(x_{0}, x_{r}\right)-\phi\left(x_{r}, x_{r}\right)<0,
$$

which contradicts

$$
\left\langle u_{r}, \eta\left(x_{0}, x_{r}\right)\right\rangle+\phi\left(x_{0}, x_{r}\right)-\phi\left(x_{r}, x_{r}\right) \geq 0 .
$$


Hence there exist $r$ such that $\left\|x_{r}\right\|<r$. For any $y \in K$, we can choose $\epsilon>0$ small enough so that $\epsilon \epsilon(0,1)$ and $u_{r}+\epsilon(y-$ $\left.x_{r}\right) \in K \cap B_{r}$. It follows from (ii) that

$$
\begin{gathered}
\left\langle u_{r}, \eta\left(x_{r}+\epsilon\left(y-x_{r}\right), x_{r}\right)\right\rangle+\phi\left(x_{r}+\epsilon\left(y-x_{r}\right), x_{r}\right) \\
-\phi\left(x_{r}, x_{r}\right) \geq 0, \quad \forall y \in K, \forall u_{r} \in T\left(x_{r}\right) .
\end{gathered}
$$

By the assumption of $\eta$, we have

$$
\begin{aligned}
&\left\langle u_{r}, \eta\left(y, x_{r}\right)\right\rangle+\phi\left(y, x_{r}\right)-\phi\left(x_{r}, x_{r}\right) \geq 0, \\
& \forall y \in K, \forall u_{r} \in T\left(x_{r}\right) .
\end{aligned}
$$

So $x_{r} \in K$ is a solution of (16).

It is easy to see that weakly relaxed $\eta-\alpha$ monotonicity implies weakly relaxed $\eta$ - $\alpha$ pseudomonotonicity. So Theorems 12,13 , and 14 are deduced to the following corollaries.

Corollary 15. Suppose that $T: K \rightarrow 2^{E^{*}}$ is $\eta$-hemicontinuous and weakly relaxed $\eta$ - $\alpha$ monotone mapping. Let $\phi: K \times K \rightarrow$ $\mathbb{R} \bigcup\{+\infty\}$ be a proper convex function and $\eta: K \times K \rightarrow E$ be a mapping. Suppose that the following conditions hold:

(i) $\eta(x, x)=0, \forall x \in K$.

(ii) For any fixed $y \in K, u \in T(x)$, the mapping $x \rightarrow$ $\langle u, \eta(x, y)\rangle$ is convex.

(iii) $x \rightarrow \eta(x, \cdot)$ and $x \rightarrow f(x, \cdot)$ are convex.

Then problems $(* * *)$ and $(* * * *)$ are equivalent as follows:

Find $x \in K$ such that

$$
\begin{aligned}
& \langle u, \eta(y, x)\rangle+\phi(y, x)-\phi(x, x) \geq 0, \\
& \forall y \in K, u \in T(x), \\
& \text { Find } x \in K \text { such that } \\
& \langle v, \eta(y, x)\rangle+\phi(y, x)-\phi(x, x) \geq \alpha(y-x), \quad(* * * *) \\
& \forall y \in K, v \in T(y) \text {. }
\end{aligned}
$$

Corollary 16. Let $K$ be a nonempty bounded closed convex subset of a real reflexive Banach space $E$ and $E^{*}$ the dual space of E. Suppose that $T: K \rightarrow 2^{E^{*}}$ is an $\eta$-hemicontinuous and weakly relaxed $\eta-\alpha$ monotone mapping. Let $\phi: K \times K \rightarrow$ $\mathbb{R} \bigcup\{+\infty\}$ be a proper convex lower semicontinuous function and $\eta: K \times K \rightarrow E$ be a mapping. Assume that

(i) $\eta(x, y)+\eta(y, x)=0, \forall x \in K$,

(ii) for any fixed $y \in K$ and $u \in T(x)$ the mapping $x \mapsto\langle u, \eta(y, x)\rangle$ is convex and lower semicontinuous function,

(iii) $x \rightarrow \eta(x, \cdot)$ and $x \rightarrow f(x, \cdot)$ are convex and lower semicontinuous,

(iv) $\alpha: E \rightarrow \mathbb{R}$ is weakly lower semicontinuous; that is, for any net $\left\{x_{\beta}\right\}, x_{\beta}$ converges to $x$ in $\sigma\left(E, E^{*}\right)$ implying that $\alpha(x) \leq \lim \inf \alpha\left(x_{\beta}\right)$.

Then problem (17) is solvable.
Corollary 17. Let $K$ be a nonempty unbounded closed convex subset of a real reflexive Banach space $E$ and $E^{*}$ the dual space of E. Suppose that $T: K \rightarrow 2^{E^{*}}$ is an $\eta$-hemicontinuous and weakly relaxed $\eta-\alpha$ monotone mapping. Let $\phi: K \times K \rightarrow$ $\mathbb{R} \bigcup\{+\infty\}$ be a proper convex lower semicontinuous function and $\eta: K \times K \rightarrow E$ be a mapping. Assume that

(i) $\eta(x, y)+\eta(y, x)=0, \forall x \in K$,

(ii) for any fixed $y \in K$ and $u \in T(x)$ the mapping $x \mapsto\langle u, \eta(y, x)\rangle$ is convex and lower semicontinuous function,

(iii) $x \rightarrow \eta(x, \cdot)$ and $x \rightarrow f(x, \cdot)$ are convex and lower semicontinuous,

(iv) $\alpha: E \rightarrow \mathbb{R}$ is weakly lower semicontinuous,

(v) $T$ is $\eta$-coercive with respect to $\phi$; that is, there exist $x_{0} \in$ $K$ such that

$$
\frac{\left\langle u-u_{0}, \eta\left(x, x_{0}\right)\right\rangle+\phi\left(x, x_{0}\right)-\phi\left(x_{0}, x_{0}\right)}{\left\|\eta\left(x, x_{0}\right)\right\|} \longrightarrow+\infty,
$$

whenever $\|x\| \rightarrow \infty$.

Then problem (16) is solvable.

Remark 18. Theorems 12, 13, and 14, improve Theorems 11, 12, and 15 of results of Kutbi and Sintunavarat [15] and also Fang and Huang [4]. These results are also the extensions of the known results of Bai et al. [1] and Hartman and Stampacchia [7] and corresponding results of Goeleven and Motreanu [5], B. S. Lee and B. D. Lee [12], Siddiqi et al. [8], and Verma [9].

\section{Competing Interests}

The authors declare that there is no conflict of interests regarding the publication of this paper.

\section{References}

[1] M.-R. Bai, S.-Z. Zhou, and G.-Y. Ni, "Variational-like inequalities with relaxed $\eta-\alpha$ pseudomonotone mappings in Banach spaces," Applied Mathematics Letters, vol. 19, no. 6, pp. 547-554, 2006.

[2] L. C. Ceng and J. C. Yao, "On generalized variational-like inequalities with generalized monotone multivalued mappings," Applied Mathematics Letters, vol. 22, no. 3, pp. 428-434, 2009.

[3] R. W. Cottle and J. C. Yao, "Pseudo-monotone complementarity problems in Hilbert space," Journal of Optimization Theory and Applications, vol. 75, no. 2, pp. 281-295, 1992.

[4] Y. P. Fang and N. J. Huang, "Variational-like inequalities with generalized monotone mappings in Banach spaces," Journal of Optimization Theory and Applications, vol. 118, no. 2, pp. 327338, 2003.

[5] D. Goeleven and D. Motreanu, "Eigenvalue and dynamic problems for variational and hemivariational inequalities," Communications on Applied Nonlinear Analysis, vol. 3, no. 4, pp. 1-21, 1996.

[6] N. Hadjisavvas and S. Schaible, "Quasimonotone variational inequalities in Banach spaces," Journal of Optimization Theory and Applications, vol. 90, no. 1, pp. 95-111, 1996. 
[7] P. Hartman and G. Stampacchia, "On some non-linear elliptic differential-functional equations," Acta Mathematica, vol. 115, pp. 271-310, 1966.

[8] A. H. Siddiqi, Q. H. Ansari, and K. R. Kazmi, "On nonlinear variational inequalities," Indian Journal of Pure and Applied Mathematics, vol. 25, no. 9, pp. 969-973, 1994.

[9] R. U. Verma, "On generalized variational inequalities involving relaxed Lipschitz and relaxed monotone operators," Journal of Mathematical Analysis and Applications, vol. 213, no. 1, pp. 387392, 1997.

[10] R. U. Verma, "Nonlinear variational inequalities on convex subsets of Banach spaces," Applied Mathematics Letters, vol. 10, no. 4, pp. 25-27, 1997.

[11] R. U. Verma, "On monotone nonlinear variational inequality problems," Commentationes Mathematicae Universitatis Carolinae, vol. 39, no. 1, pp. 91-98, 1998.

[12] B. S. Lee and B. D. Lee, "Weakly relaxed $\alpha$-semi pseudomonotone setvalued variational-like inequalities," Journal of the Korea Society of Mathematical Education, Series B: Pure and Applied Mathematics, vol. 11, no. 3, pp. 231-241, 2004.

[13] M. A. Kang, N. J. Huang, and B. S. Lee, "Generalized pseudomonotone setvalued variational-like inequalities," Indian Journal of Mathematics, vol. 45, no. 3, pp. 251-264, 2003.

[14] W. Sintunavarat, "Mixed equilibrium problems with weakly relaxed $\alpha$-monotone bifunction in banach spaces," Journal of Function Spaces and Applications, vol. 2013, Article ID 374268, 5 pages, 2013.

[15] M. A. Kutbi and W. Sintunavarat, "On the solution existence of variational-like inequalities problem for weakly relaxed $\eta-\alpha$ monotone mapping," Abstract and Applied Analysis, vol. 2013, Article ID 207845, 8 pages, 2013.

[16] B. Knaster, C. Kuratowski, and S. Mazurkiewicz, "Ein Beweis des Fixpunktsatzes fur n-dimensionale Simplexe," Fundamenta Mathematicae, vol. 14, no. 1, pp. 132-137, 1929.

[17] J. C. Yao, "Existence of generalized variational inequalities," Operations Research Letters, vol. 15, no. 1, pp. 35-40, 1994.

[18] X. Q. Yang and G. Y. Chen, "A class of nonconvex functions and pre-variational inequalities," Journal of Mathematical Analysis and Applications, vol. 169, no. 2, pp. 359-373, 1992.

[19] K. Fan, "A generalization of Tychonoff's fixed point theorem," Mathematische Annalen, vol. 142, pp. 305-310, 1961. 


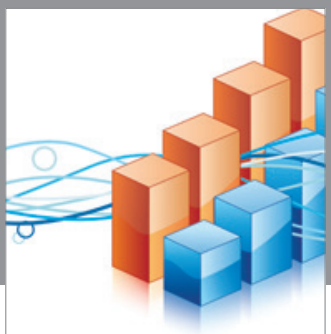

Advances in

Operations Research

vatem alat4

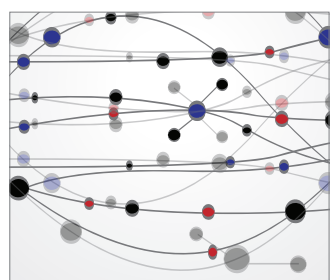

\section{The Scientific} World Journal
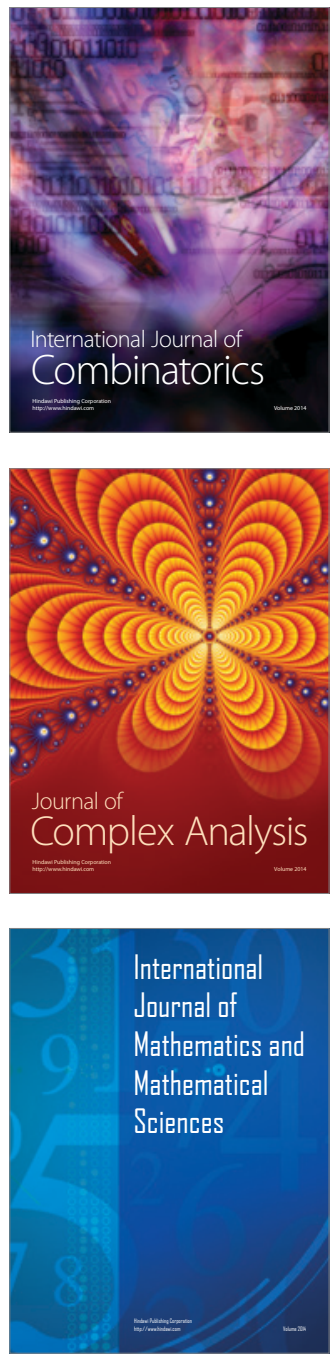
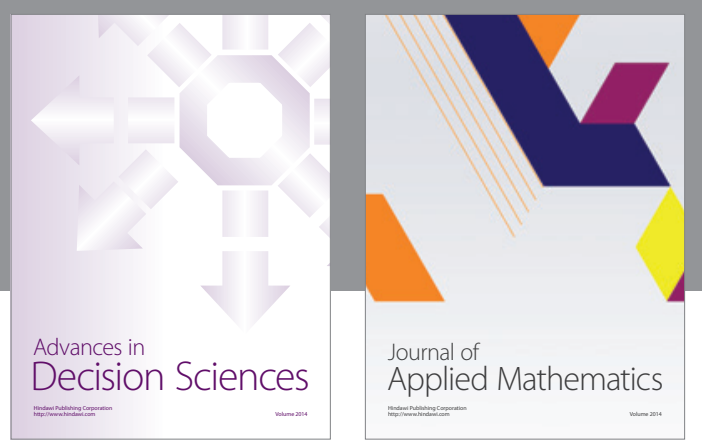

Algebra

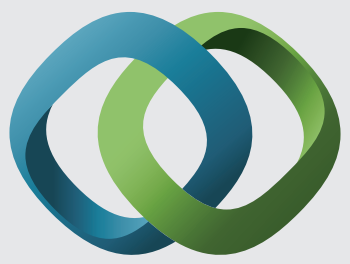

\section{Hindawi}

Submit your manuscripts at

http://www.hindawi.com
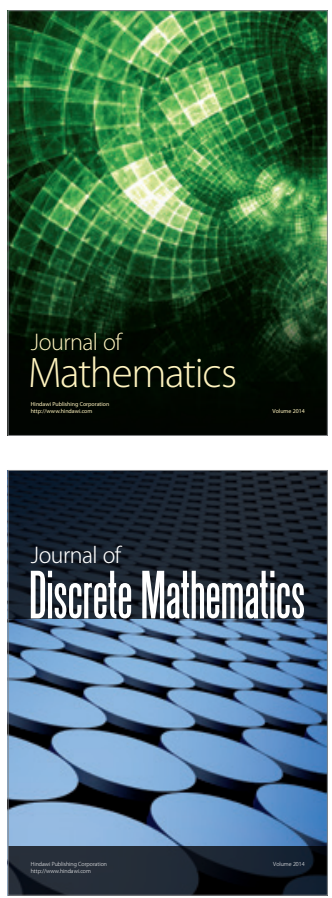

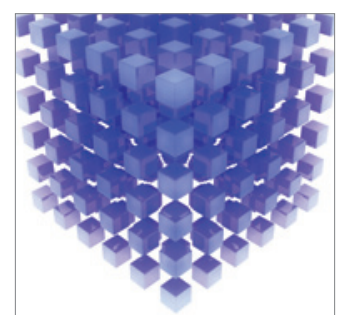

Mathematical Problems in Engineering
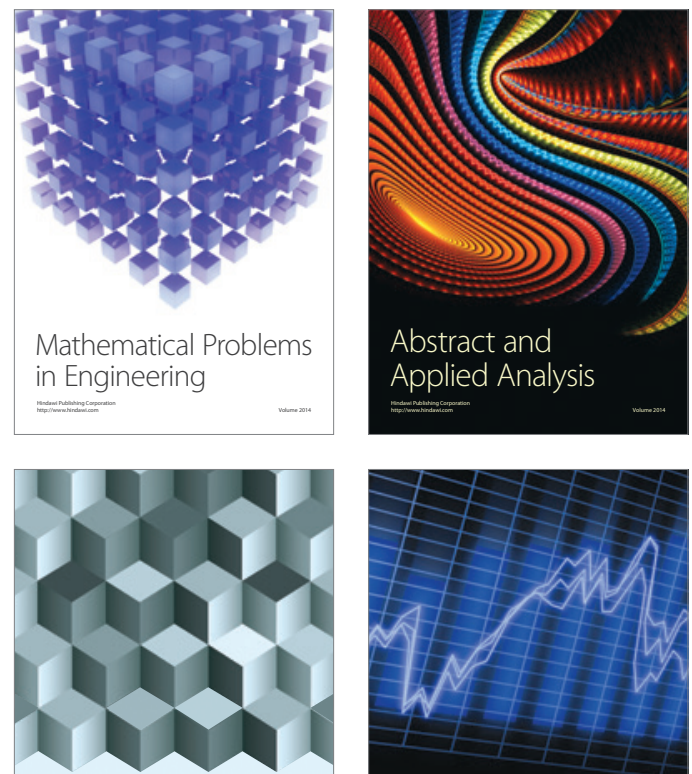

Journal of

Function Spaces

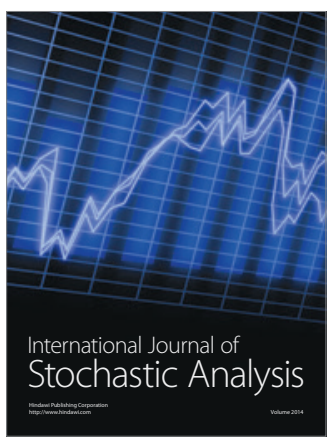

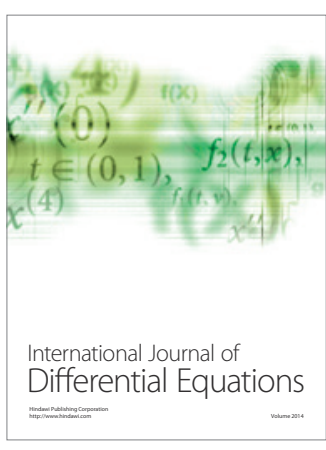
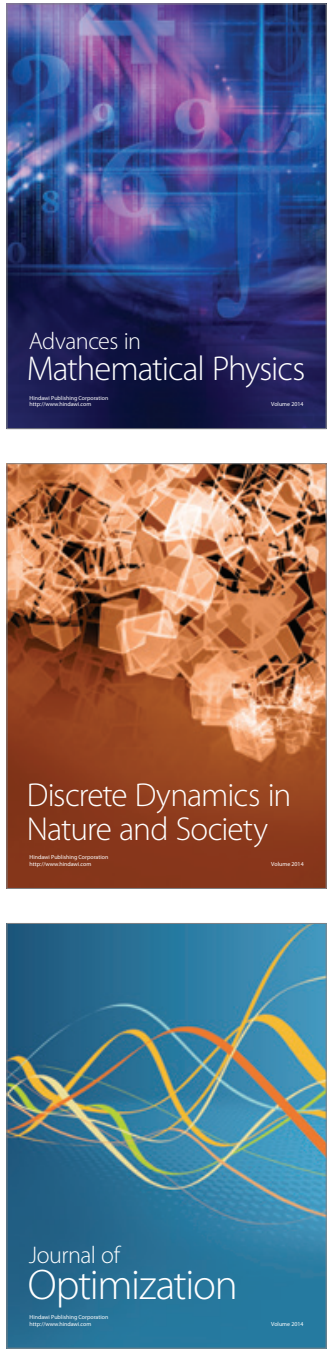